\title{
On the Liouville theorem for weak Beltrami flows
}

\author{
Dongho Chae* and Jörg Wolf ${ }^{\dagger}$ \\ *Department of Mathematics \\ Chung-Ang University \\ Seoul 156-756, Republic of Korea \\ e-mail: dchae@cau.ac.kr \\ and \\ $\dagger$ Department of Mathematics \\ Humboldt University Berlin \\ Unter den Linden 6, 10099 Berlin, Germany \\ e-mail: jwolf@math.hu-berlin.de
}

\begin{abstract}
We study Beltrami flows in the setting of weak solution to the stationary Euler equations in $\mathbb{R}^{3}$. For this weak Beltrami flow we prove the regularity and the Liouville property. In particular, we show that if tangential part of the velocity has certain decay property at infinity, then the solution becomes trivial. This decay condition of of the velocity is weaker than the previously known sufficient conditions for the Liouville property of the Betrami flows. For the proof we establish a mean value formula and other various formula for the tangential and the normal components of the weak solutions to the stationary Euler equations.
\end{abstract}

AMS Subject Classification Number: 76B03, 35Q31

keywords: Euler's equations, Beltrami flows, Liouville's theorem

\section{Introduction}

We consider the stationary Euler equation in $\mathbb{R}^{3}$

$$
(\boldsymbol{u} \cdot \nabla) \boldsymbol{u}=-\nabla p, \quad \nabla \cdot \boldsymbol{u}=0,
$$

where $\boldsymbol{u}=\left(u_{1}(x), u_{2}(x), u_{3}(x)\right), x \in \mathbb{R}^{3}$. Together with the Navier-Stokes equations the Euler system is of fundamental importance in the fluid mechanics. Even with such case the stationary Euler equations is less well studied than the Navier-Stokes equations. On the other hand the Euler equations have rich geometric structure as can be seen in the book by Arnold and Khesin([1]) for example. Using the vector identity

$$
\frac{1}{2} \nabla|\boldsymbol{u}|^{2}=(\boldsymbol{u} \cdot \nabla) \boldsymbol{u}+\boldsymbol{u} \times(\nabla \times \boldsymbol{u})
$$


one can rewrite the system (1.1) as

$$
\boldsymbol{u} \times \omega=\nabla\left(p+\frac{1}{2}|\boldsymbol{u}|^{2}\right), \quad \omega=\nabla \times \boldsymbol{u} .
$$

A vector field in $\mathbb{R}^{3}, \boldsymbol{u}$ is called the Beltrami field if there exists a scalar function $\lambda=\lambda(x)$ such that $\varnothing=\lambda \boldsymbol{u}$. If $\boldsymbol{u}$ is a Betrami field, and simultaneously is a solution to (1.1), then the pressure should be given by $p=-\frac{1}{2}|\boldsymbol{u}|^{2}$ up to addition of a constant. Conversely, if $(\boldsymbol{u}, p)$ solves (1.1) with $p=-\frac{1}{2}|\boldsymbol{u}|^{2}$, then $\boldsymbol{u}$ is a Beltrami field. A Beltrami field, which is a solution to the stationary Euler system, is called the Beltrami flow. The Beltrami fields has special roles in the physics of turbulence. In fact it is observed numerically in [8] that turbulent flow consists of the superposition of the Beltrami fields. This aspect was theoretically studied in [4], and more recently the Beltrami fields are crucially used to construct energy dissipating continuous weak solutions of the time dependent Euler equations([5]). For the study of Beltrami flows the authors of [6] constructed a non-trivial solution $\boldsymbol{u}(x)$ which decays of $O\left(\frac{1}{|x|}\right)$ as $|x| \rightarrow+\infty$. Remarkably enough, in [7] it is shown later that if either the Beltrami flow $\boldsymbol{u}$ decays like $o\left(\frac{1}{|x|}\right)$ as $|x| \rightarrow+\infty$ or $\boldsymbol{u} \in L^{q}\left(\mathbb{R}^{3}\right), q \in[2,3]$, then $\boldsymbol{u} \equiv 0$. Another sufficient condition for this Liouville type property $\frac{|\boldsymbol{u}|^{2}}{|\boldsymbol{x}|^{\mu}} \in L^{1}\left(\mathbb{R}^{3}\right), \mu \in[0,1]$ is also found in [3]. These studies on the Beltrami flow was in the setting of classical formulation, assuming $C^{1}\left(\mathbb{R}^{3}\right)$ regularity or higher for the velocity fields. In this paper we formulate the notion of the Beltrami flow in the weak sense, and study its regularity as well as the Liouville type property. Based on the mean value formula as derived in the next section we could obtain, among others, much weaker condition for the Liouville property, imposing the decay condition only on the tangential component of the velocity.

We start from the weak formulation of (1.1).

Definition 1.1. A pair $(\boldsymbol{u}, p) \in\left[L_{\mathrm{loc}}^{2}\left(\mathbb{R}^{3}\right)\right]^{3} \times L_{\text {loc }}^{1}\left(\mathbb{R}^{3}\right)$ is said to be a weak solution to (1.1) if

$$
\int_{\mathbb{R}^{3}} \boldsymbol{u} \otimes \boldsymbol{u}: \nabla \boldsymbol{\varphi} d x=-\int_{\mathbb{R}^{3}} p \nabla \cdot \boldsymbol{\varphi} d x \quad \forall \boldsymbol{\varphi} \in\left[C_{\mathrm{c}}^{\infty}\left(\mathbb{R}^{3}\right)\right]^{3}
$$

Then, we can generalize the classical notion of the Beltrami flow as follows.

Definition 1.2. A weak solution $(\boldsymbol{u}, p)$ to (1.1) is called weak Beltrami flow if it satisfies

$$
p=-\frac{|\boldsymbol{u}|^{2}}{2} \quad \text { a.e. in } \quad \mathbb{R}^{3}
$$

We denote the normal and the tangential part of the velocity $\boldsymbol{u}(x)$ are defined respectively as follows.

$$
\boldsymbol{u}_{N}:=\left(\boldsymbol{u} \cdot \frac{x}{|x|}\right) \frac{x}{|x|}, \quad \boldsymbol{u}_{T}:=\boldsymbol{u} \times \frac{x}{|x|},
$$


where the equality holds almost everywhere in $\mathbb{R}^{3}$. Then, we have the orthogonal decomposition for any vector field on $\mathbb{R}^{3}$.

$$
\boldsymbol{u}=\boldsymbol{u}_{N}+\boldsymbol{u}_{T}
$$

The main purpose of this paper is to present decay conditions on $\boldsymbol{u}_{T}=\boldsymbol{u} \times \frac{x}{|x|}$, which imply the Liouville property $\boldsymbol{u} \equiv \mathbf{0}$ for the weak Beltrami flows. This would extend the previous Liouville type results in [7, 3]. In addition, we show that for a weak Beltrami flow the velocity in fact enjoys certain regularity properties in terms of weighted Lebesgue spaces. Both results will be achieved from various identities involving the tangential part and the normal part of $\boldsymbol{u}$.

We first state the following Liouville property for a weak Beltrami flow.

Theorem 1.3. Let $\boldsymbol{u} \in \boldsymbol{L}_{\text {loc }}^{2}$ be a weak Beltrami flow. Then

$$
\int_{B_{R}} \frac{\left|\boldsymbol{u}_{N}\right|^{2}}{|x|} d x \leq \frac{1}{2 R} \int_{B_{R}}|\boldsymbol{u}|^{2} d x=\frac{1}{2} \int_{\partial B_{R}}\left(\left|\boldsymbol{u}_{T}\right|^{2}-\left|\boldsymbol{u}_{N}\right|^{2}\right) d S \quad \forall 0<R<+\infty .
$$

Therefore, if there exists a sequence $R_{k} \rightarrow+\infty$ such that

$$
\int_{\partial B_{R_{k}}}\left|\boldsymbol{u}_{T}\right|^{2} d S \rightarrow 0 \quad \text { as } \quad k \rightarrow+\infty
$$

then $\boldsymbol{u} \equiv 0$.

Remark 1.4. As immediate consequences of the above theorem we obtain the previously known Liouville type results in [7, 3]. Indeed, let $\boldsymbol{u} \in \boldsymbol{L}_{\text {loc }}^{2}$ be a weak Beltrami flow, satisfying one of the following conditions.

(i) $\left|\boldsymbol{u}_{T}(x)\right|=o\left(|x|^{-1}\right)$ as $|x| \rightarrow+\infty$.

(ii) $\boldsymbol{u}_{T} \in\left[L^{q}\left(\mathbb{R}^{3}\right)\right]^{3}$ for some $q \in[2,3]$.

(iii) $\frac{\left|\boldsymbol{u}_{T}\right|^{2}}{|x|^{\mu}} \in L^{1}\left(\mathbb{R}^{3}\right)$ for some $\mu \in(-\infty, 1]$.

Then, one can find a sequence $\left\{R_{k}\right\}_{k \in \mathbb{N}}$ such that (1.7) holds. The case (i) is obvious. In the case (ii) from the condition

$$
\left\|\boldsymbol{u}_{T}\right\|_{L^{q}}^{q}=\int_{0}^{+\infty} \int_{\partial B_{R}}\left|\boldsymbol{u}_{T}\right|^{q} d S d R<+\infty
$$

one can deduce that there exists a sequence $\left\{R_{k}\right\}_{k \in \mathbb{N}}$ such that

$$
\int_{\partial B_{R_{k}}}\left|\boldsymbol{u}_{T}\right|^{q} d S=o\left(\frac{1}{R_{k}}\right) \text { as } k \rightarrow+\infty .
$$

Therefore, for each $q \in(2,3]$, we obtain

$$
\int_{\partial B_{R_{k}}}\left|\boldsymbol{u}_{T}\right|^{2} d S \leq C\left(\int_{\partial B_{R_{k}}}\left|\boldsymbol{u}_{T}\right|^{q} d S\right)^{\frac{2}{q}} R_{k}^{\frac{2(q-2)}{q}}=o\left(R_{k}^{-\frac{2}{q}}\right) R_{k}^{\frac{2(q-2)}{q}}=o(1) \quad \text { as } \quad k \rightarrow+\infty .
$$


In the case (iii) we have for $\mu \in(-\infty, 1]$

$$
\int_{0}^{+\infty} R^{-\mu} \int_{\partial B_{R}}\left|\boldsymbol{u}_{T}\right|^{2} d S d R<+\infty
$$

from which we find there exists a sequence $\left\{R_{k}\right\}_{k \in \mathbb{N}}$ such that

$$
\int_{\partial B_{R_{k}}}\left|\boldsymbol{u}_{T}\right|^{2} d S=o\left(\frac{1}{R_{k}}\right) R_{k}^{\mu}=o(1) \quad \text { as } \quad k \rightarrow+\infty .
$$

Remark 1.5. If $\boldsymbol{u}$ is a non-trivial Beltrami flow such that $|\boldsymbol{u}| \leq \frac{K}{|x|}(K=$ const $>0)$, as has been constructed in [6] for example, then

$$
\int_{\mathbb{R}^{3}} \frac{\left|\boldsymbol{u}_{T}\right|^{2}}{|x|} d x=+\infty, \quad \text { and } \int_{\mathbb{R}^{3}} \frac{\left|\boldsymbol{u}_{N}\right|^{2}}{|x|} d x<+\infty .
$$

Hence, $\boldsymbol{u}_{N}$ has stronger decay then $\boldsymbol{u}_{T}$. Clearly the first statement follows directly from (iii) of Remark 1.4, while the second one follows from (1.6) together with

$$
\int_{B_{R}} \frac{\left|\boldsymbol{u}_{N}\right|^{2}}{|x|} d x \leq \frac{1}{2} \int_{\partial B_{R}}\left(\left|\boldsymbol{u}_{T}\right|^{2}-\left|\boldsymbol{u}_{N}\right|^{2}\right) d S \leq 2 \pi K^{2} \quad \forall 0<R<+\infty,
$$

and passing $R \rightarrow+\infty$.

Let us recall that we say a vector field $\boldsymbol{u} \in\left[L_{\text {loc }}^{p}\left(\mathbb{R}^{3}\right)\right]^{3}, 1 \leq p \leq+\infty$, belongs to the local Morrey space $\left[L_{\text {loc }}^{p, \lambda}\left(\mathbb{R}^{3}\right)\right]^{3}$ if

$$
\sup _{\rho>0}\left(\rho^{-\lambda} \int_{B_{\rho}}|\boldsymbol{u}|^{p} d x\right)<+\infty .
$$

The following is a regularity result for weak Beltrami flows.

Theorem 1.6. Let $\boldsymbol{u} \in \boldsymbol{L}_{\mathrm{loc}}^{2}$ be a weak Beltrami flow. Then, $\boldsymbol{u} \in\left[L_{\mathrm{loc}}^{2,1}\left(\mathbb{R}^{3}\right)\right]^{3}$.

\section{Mean value formulas for weak solutions to the stationary Euler equation}

The aim of this section is to derive identities obtained from (1.4). We have the following

Lemma 2.1. Let $(\boldsymbol{u}, p)$ be a weak solution to the stationary Euler equation. Then there holds

$$
\int_{\partial B_{R}}\left(p+\left|\boldsymbol{u}_{N}\right|^{2}\right) d S=\frac{1}{R} \int_{B_{R}}\left(3 p+|\boldsymbol{u}|^{2}\right) d x \quad \text { for a. e. } 0<R<+\infty \text {. }
$$


Remark 2.2. A formula similar to (2.1) is derived in Theorem 1.2 of [2], where the integration over $B_{R}$ is replaced by the integration over $\mathbb{R}^{3} \backslash B_{R}$. In the above lemma our assumptions on $(\boldsymbol{u}, p)$ are much weaker in terms of the regularity and the integrability, and the method of proof given below is completely different from [2].

Proof of Lemma 2.1 We first regularize the Euler equations, using the standard mollifying kernel $\rho_{\varepsilon}=\varepsilon^{-3} \rho\left(\varepsilon^{-1} x\right), 1<\varepsilon<+\infty$, where $\rho \in C_{\mathrm{c}}^{\infty}\left(B_{1}\right)$ is nonnegative, and radial symmetric together with the normalized condition

$$
\int_{\mathbb{R}^{3}} \rho d x=1 .
$$

For $f \in L_{\text {loc }}^{1}\left(\mathbb{R}^{3}\right)$ we define

$$
f_{\varepsilon}(x)=\int_{\mathbb{R}^{3}} f(y) \rho_{\varepsilon}(x-y) d y, \quad x \in \mathbb{R}^{3} .
$$

We note that (2.2) implies

$$
\int_{B_{R}}\left|f_{\varepsilon}\right| d x \leq \int_{B_{R+\varepsilon}}|f| d x \quad \forall 0<R<+\infty .
$$

From (1.4) with $\varphi_{\varepsilon}$ in place of $\boldsymbol{\varphi}$ we easily see that

$$
\nabla \cdot(\boldsymbol{u} \otimes \boldsymbol{u})_{\varepsilon}=-\nabla p_{\varepsilon} \quad \text { in } \quad \mathbb{R}^{3} .
$$

We multiply both sides of (2.4) by $x$, and for $0<r<+\infty$ we integrate the result over $\partial B_{r}$ with respect to the two dimensional surface measure. This yields

$$
\int_{\partial B_{r}} \partial_{j}\left(u^{j} u^{i}\right)_{\varepsilon} x_{i} d S=-\int_{\partial B_{r}} \partial_{i} p_{\varepsilon} x_{i} d S,
$$

where and hereafter we use the Einstein convention that the repeated indices imply the summation over $\{1,2,3\}$. Let $-\infty<\alpha<3$. We multiply both sides of this by $r^{-\alpha}=|x|^{-\alpha}$, and integrate it over $(0, R), 0<R<+\infty$ with respect to $r$, and then apply the integration by parts. Thus, we have

$$
\begin{aligned}
\int_{B_{R}} \partial_{j}\left(u^{j} u^{i}\right)_{\varepsilon} x_{i}|x|^{-\alpha} d x & =-\int_{B_{R}}\left(|\boldsymbol{u}|^{2}\right)_{\varepsilon}|x|^{-\alpha} d x+\alpha \int_{B_{R}}\left(u^{i} u^{j}\right)_{\varepsilon} x_{i} x_{j}|x|^{-\alpha-2} d x \\
& \quad+\int_{\partial B_{R}}\left(u^{i} u^{j}\right)_{\varepsilon} x_{i} x_{j}|x|^{-\alpha-1} d S \\
& =-\int_{B_{R}} \partial_{i} p_{\varepsilon} x_{i}|x|^{-\alpha} d x \\
& =(3-\alpha) \int_{B_{R}} p_{\varepsilon}|x|^{-\alpha} d x-\int_{\partial B_{R}} p_{\varepsilon}|x|^{-\alpha+1} d S .
\end{aligned}
$$


From the above identity after a routine manipulation we obtain

$$
R^{1-\alpha} \int_{\partial B_{R}}\left(p_{\varepsilon}+v_{N, \varepsilon}\right) d S=\int_{B_{R}}\left\{(3-\alpha) p_{\varepsilon}+\left(|\boldsymbol{u}|^{2}\right)_{\varepsilon}-\alpha v_{N, \varepsilon}\right\}|x|^{-\alpha} d x,
$$

where we have set

$$
\begin{aligned}
v_{N, \varepsilon}(x) & =\left(u^{i} u^{j}\right)_{\varepsilon} \frac{x_{i} x_{j}}{|x|^{2}}=\frac{1}{|x|^{2}} \int_{\mathbb{R}^{3}}(\boldsymbol{u}(y) \cdot x)^{2} \rho_{\varepsilon}(x-y) d y \\
& =\int_{\mathbb{R}^{3}}\left|\boldsymbol{u}_{N}(x)\right|^{2} \rho_{\varepsilon}(x-y) d y, \quad \forall x \in \mathbb{R}^{3} \backslash\{0\} .
\end{aligned}
$$

In particular, (2.6) with $\alpha=0$ yields

$$
\int_{\partial B_{r}}\left(p_{\varepsilon}+v_{N, \varepsilon}\right) d S=\frac{1}{r} \int_{B_{r}} 3 p_{\varepsilon}+\left(|\boldsymbol{u}|^{2}\right)_{\varepsilon} d x \quad \forall 0 \leq r<+\infty .
$$

For an arbitrary $h>0$ we integrate the above identity over $(R, R+h)$, and then letting $\varepsilon \rightarrow 0$. This leads to

$$
\frac{1}{h} \int_{R}^{R+h} \int_{\partial B_{r}}\left(p+\left|\boldsymbol{u}_{N}\right|^{2}\right) d S d r=\frac{1}{h} \int_{B_{R+h} \backslash B_{R}}\left(p+\left|\boldsymbol{u}_{N}\right|^{2}\right) d x=\frac{1}{h} \int_{R}^{R+h} \frac{1}{r} \int_{B_{r}}\left(3 p+|\boldsymbol{u}|^{2}\right) d x d r .
$$

Since $r \mapsto \int_{\partial B_{r}}\left(p+\left|\boldsymbol{u}_{N}\right|^{2}\right) d S$ is $L_{\text {loc }}^{1}([0,+\infty))$, we have

$$
\lim _{h \rightarrow 0} \frac{1}{h} \int_{R}^{R+h} \int_{\partial B_{r}}\left(p+\left|\boldsymbol{u}_{N}\right|^{2}\right) d S d r=\int_{\partial B_{R}}\left(p+\left|\boldsymbol{u}_{N}\right|^{2}\right) d S \quad \text { for a. e. } 0<R<+\infty .
$$

Hence, the assertion follows from (2.9) after letting $h \rightarrow 0$ on both sides.

Remark 2.3. Define,

$$
\varphi(r)=-2 \int_{\partial B_{r}}\left(p+\left|\boldsymbol{u}_{N}\right|^{2}\right) d S, \quad 0<r<+\infty .
$$

By virtue of (2.9) we see that each $r \in(0,+\infty)$ is a Lebesgue point of $\varphi$. Therefore $\varphi$ can be identified with the continuous function $r \mapsto-\frac{2}{r} \int_{B_{r}}\left(3 p+|\boldsymbol{u}|^{2}\right) d x$, which is actually absolutely continuous. Indeed, let us set $-2\left(3 p+|\boldsymbol{u}|^{2}\right):=g \in L_{\mathrm{loc}}^{1}\left(\mathbb{R}^{3}\right)$, and $\left\{\left(a_{i}, b_{i}\right)\right\}_{i \in J}$ be a collection of non-overlapping intervals such that $0<r=\inf _{i \in J} a_{i}$, and $R=\sup _{i \in J} b_{i}<+\infty$. Then,

$$
\begin{array}{r}
\sum_{i \in J}\left|\varphi\left(b_{i}\right)-\varphi\left(a_{i}\right)\right|=\sum_{i \in J}\left|\frac{1}{b_{i}} \int_{B_{b_{i}} \backslash B_{a_{i}}} g d x-\frac{b_{i}-a_{i}}{a_{i} b_{i}} \int_{B_{a_{i}}} g d x\right| \\
\leq \frac{1}{r} \sum_{i \in J} \int_{B_{b_{i} \backslash B_{a_{i}}}|g| d x+\frac{1}{r^{2}} \sum_{i \in J}\left(b_{i}-a_{i}\right) \int_{B_{R}}|g| d x \rightarrow 0}
\end{array}
$$


as $\sum_{i \in J}\left(b_{i}-a_{i}\right) \rightarrow 0$. By the similar argument we have used in the proof of Lemma2.1, from (2.6) we claim that the following general identity holds true.

$$
R^{1-\alpha} \varphi(R)-r^{1-\alpha} \varphi(r)=-2 \int_{B_{R} \backslash B_{r}}\left\{(3-\alpha) p+|\boldsymbol{u}|^{2}-\alpha\left|\boldsymbol{u}_{N}\right|^{2}\right\}|x|^{-\alpha} d x
$$

for all $0<r<R$ and $\alpha \in \mathbb{R}$. Indeed, given $0<\sigma<\rho$ we multiply both sides of (2.5) (with $0<\varepsilon<\sigma$ ) by $r^{-\alpha}=|x|^{-\alpha}$. Then we integrate the result over $(\sigma, \rho)$, and integrate by parts. This yields

$$
\begin{aligned}
\int_{B_{\rho} \backslash B_{\sigma}} & \partial_{j}\left(u^{j} u^{i}\right)_{\varepsilon} x_{i}|x|^{-\alpha} d x \\
= & -\int_{B_{\rho} \backslash B_{\sigma}}\left(|\boldsymbol{u}|^{2}\right)_{\varepsilon}|x|^{-\alpha} d x+\alpha \int_{B_{\rho} \backslash B_{\sigma}}\left(u^{i} u^{j}\right)_{\varepsilon} x_{i} x_{j}|x|^{-\alpha-2} d x \\
& \quad+\int_{\partial B_{\rho}}\left(u^{i} u^{j}\right)_{\varepsilon} x_{i} x_{j}|x|^{-\alpha-1} d S-\int_{\partial B_{\sigma}}\left(u^{i} u^{j}\right)_{\varepsilon} x_{i} x_{j}|x|^{-\alpha-1} d S \\
=-\int_{B_{\rho} \backslash B_{\sigma}} \partial_{i} p_{\varepsilon} x_{i}|x|^{-\alpha} d x & (3-\alpha) \int_{B_{\rho} \backslash B_{\sigma}} p_{\varepsilon}|x|^{-\alpha} d x-\int_{\partial B_{\rho}} p_{\varepsilon}|x|^{-\alpha+1} d S+\int_{\partial B_{\sigma}} p_{\varepsilon}|x|^{-\alpha+1} d S .
\end{aligned}
$$

Accordingly,

$$
\begin{aligned}
\rho^{1-\alpha} \int_{\partial B_{\rho}}\left(p_{\varepsilon}+v_{N, \varepsilon}\right) d S-\sigma^{1-\alpha} \int_{\partial B_{\sigma}}\left(p_{\varepsilon}+v_{N, \varepsilon}\right) d S \\
=\int_{B_{\rho} \backslash B_{\sigma}}\left\{(3-\alpha) p_{\varepsilon}+\left(|\boldsymbol{u}|^{2}\right)_{\varepsilon}-\alpha v_{N, \varepsilon}\right\}|x|^{-\alpha} d x .
\end{aligned}
$$

Let $0<r<R$ be arbitrarily chosen and let $0<\varepsilon<r$. Given $0<h<r-\varepsilon$, we intgrate (2.12) first over $(R, R+h)$ with respect to $\rho$ and second over $(r, r+h)$ with respect to $\sigma$. Then dividing both sides by $h^{2}$, we have

$$
\begin{aligned}
\frac{1}{h} \int_{R}^{R+h} \rho^{1-\alpha} \int_{\partial B_{\rho}}\left(p_{\varepsilon}+v_{N, \varepsilon}\right) d S d \rho-\frac{1}{h} \int_{r}^{r+h} \sigma^{1-\alpha} \int_{\partial B_{\sigma}}\left(p_{\varepsilon}+v_{N, \varepsilon}\right) d S d \sigma \\
\quad=\frac{1}{h^{2}} \int_{r}^{r+h} \int_{R}^{R+h} \int_{B_{\rho} \backslash B_{\sigma}}\left\{(3-\alpha) p_{\varepsilon}+\left(|\boldsymbol{u}|^{2}\right)_{\varepsilon}-\alpha v_{N, \varepsilon}\right\}|x|^{-\alpha} d x d \rho d \sigma
\end{aligned}
$$

where we used the notation of (2.7). Passing $\varepsilon \rightarrow 0$, using the convergence properties 
of the mollified functions, we obtain that the left hand side of (2.13) converges to

$$
\begin{gathered}
\frac{1}{h} \int_{R}^{R+h} \rho^{1-\alpha} \int_{\partial B_{\rho}}\left(p+\left|\boldsymbol{u}_{N}\right|^{2}\right) d S d \rho-\frac{1}{h} \int_{r}^{r+h} \sigma^{1-\alpha} \int_{\partial B_{\sigma}}\left(p+|\boldsymbol{u}|_{N}\right) d S d \sigma \\
=-\frac{1}{2 h} \int_{R}^{R+h} \rho^{1-\alpha} \varphi(\rho) d \rho+\frac{1}{2 h} \int_{r}^{r+h} \sigma^{1-\alpha} \varphi(\sigma) d \sigma .
\end{gathered}
$$

Passing $\varepsilon \rightarrow 0$ in the right-hand side of (2.13), we first observe that

$\int_{B_{\rho} \backslash B_{\sigma}}\left\{(3-\alpha) p_{\varepsilon}+\left(|\boldsymbol{u}|^{2}\right)_{\varepsilon}-\alpha v_{N, \varepsilon}\right\}|x|^{-\alpha} d x \rightarrow \int_{B_{\rho} \backslash B_{\sigma}}\left\{(3-\alpha) p+|\boldsymbol{u}|^{2}-\alpha\left|\boldsymbol{u}_{N}\right|^{2}\right\}|x|^{-\alpha} d x$

for each $\rho \in(R, R+h)$ and $\sigma \in(r, r+h)$. Thus, by means of Lebesgue's theorem of dominated convergence we see that the right-hand side of (2.13) tends to

$$
\frac{1}{h^{2}} \int_{r}^{r+h} \int_{R}^{R+h} \int_{B_{\rho} \backslash B_{\sigma}}\left\{(3-\alpha) p+|\boldsymbol{u}|^{2}-\alpha\left|\boldsymbol{u}_{N}\right|^{2}\right\}|x|^{-\alpha} d x d \rho d \sigma
$$

as $\varepsilon \rightarrow 0$. With the help the above limits we are able to carry out the passage to the limit $\varepsilon \rightarrow 0$ in (2.13) . Letting $h \rightarrow 0$ in both of (2.14) and (2.15) and equating them to each other, we obtain (2.11). The claim is proved.

In the case when the mean value $\left(3 p+|\boldsymbol{u}|^{2}\right)_{B_{r}}$ is bounded for $r \rightarrow 0^{+}$, it follows that $r^{-2} \varphi(r)$ is bounded too as $r \rightarrow 0^{+}$, which shows that $r^{-1-\alpha} \varphi(r) \rightarrow 0$ as $r \rightarrow 0^{+}$for all $\alpha<1$. However, this property might not be true in general.

Furthermore, from (2.11) we easily get $\varphi \in W_{\text {loc }}^{1,1}((0,+\infty))$, and there holds

$$
\varphi+R \varphi^{\prime}=-2 \int_{\partial B_{R}}\left(3 p+|\boldsymbol{u}|^{2}\right) d S \text { for a. e. } 0<R<+\infty
$$

and, substituting $\varphi$ from (2.10) into (2.16), using the fact $|\boldsymbol{u}|^{2}=\left|\boldsymbol{u}_{N}\right|^{2}+\left|\boldsymbol{u}_{T}\right|^{2}$, we arrive at

$$
\varphi^{\prime}=-\frac{2}{R} \int_{\partial B_{R}}\left(2 p+\left|\boldsymbol{u}_{T}\right|^{2}\right) d S=\frac{2}{R} \varphi+\frac{2}{R} \int_{\partial B_{R}}\left(3\left|\boldsymbol{u}_{N}\right|^{2}-|\boldsymbol{u}|^{2}\right) d S
$$

for a.e. $0<R<+\infty$.

In case of Beltrami flow, we have $2 p=-|\boldsymbol{u}|^{2}$, and we get from (2.1)

$$
\varphi(r)=\int_{\partial B_{r}}\left(\left|\boldsymbol{u}_{T}\right|^{2}-\left|\boldsymbol{u}_{N}\right|^{2}\right) d S=\frac{1}{r} \int_{B_{r}}|\boldsymbol{u}|^{2} d x \quad \forall 0<r<+\infty .
$$


This shows that $\varphi$ is nonnegative. Furthermore from (2.16) we get

$$
\varphi^{\prime}=\frac{1}{R} \int_{\partial B_{R}}|\boldsymbol{u}|^{2} d S-\frac{\varphi}{R}=\frac{2}{R} \int_{\partial B_{R}}\left|\boldsymbol{u}_{N}\right|^{2} d S \quad \text { a. e. in }(0,+\infty),
$$

and therefore $\varphi$ is nondecreasing. In particular,

$$
\exists \lim _{R \rightarrow 0^{+}} \varphi(R) \geq 0 .
$$

Proof of Theorem 1.3 Observing (2.11) with $\alpha=1$ along with $\varphi(r) \geq 0$, we infer

$$
2 \int_{B_{R} \backslash B_{r}} \frac{\left|\boldsymbol{u}_{N}\right|^{2}}{|x|} d x=\varphi(R)-\varphi(r) \leq \varphi(R) \quad \forall 0<r<R .
$$

This shows that $\frac{\left|\boldsymbol{u}_{N}\right|^{2}}{|x|} \in L_{\text {loc }}^{1}\left(\mathbb{R}^{3}\right)$ and from monotone convergence of the measure we conclude (1.6).

If the condition (1.7) is true, then from (1.6) and $\varphi(R) \leq \int_{\partial B_{R}}\left|\boldsymbol{u}_{T}\right|^{2} d S$ we get

$$
\int_{B_{R_{k}}} \frac{\left|\boldsymbol{u}_{N}\right|^{2}}{|x|} d x \leq \int_{\partial B_{R_{k}}}\left|\boldsymbol{u}_{T}\right|^{2} d S \rightarrow 0 \quad \text { as } \quad k \rightarrow+\infty .
$$

Accordingly, $\frac{\left|\boldsymbol{u}_{N}\right|^{2}}{|x|}=0$ which implies $\boldsymbol{u}_{N} \equiv 0$, and therefore $\boldsymbol{u}=\boldsymbol{u}_{T}$. On the other hand from (2.11) with $\alpha=1$ it follows that $\varphi=\varphi_{0} \equiv$ const $\geq 0$. Consequently, in view of (1.7)

$$
\varphi_{0}=\int_{\partial B_{R_{k}}}\left|\boldsymbol{u}_{T}\right|^{2} d S \rightarrow 0 \quad \text { as } \quad k \rightarrow+\infty .
$$

Whence, $\boldsymbol{u}=\boldsymbol{u}_{T} \equiv 0$.

Proof of Theorem $\mathbf{1 . 6}$ First, by the monotonicity of $\varphi$ we observe

$$
\int_{B_{2}}|\boldsymbol{u}|^{2} d x \geq \int_{B_{2}}\left(\left|\boldsymbol{u}_{T}\right|^{2}-\left|\boldsymbol{u}_{N}\right|^{2}\right) d x \geq \int_{1}^{2} \varphi(r) d r \geq \varphi(1) .
$$

By means of (2.1) we see that

$$
\frac{1}{R} \int_{B_{R}}|\boldsymbol{u}|^{2} d x \leq \varphi(R) \leq \varphi(1) \quad \forall 0<R \leq 1 .
$$

Then the assertion of the theorem follows by combining (2.21) with (2.22).

\section{Acknowledgements}

Chae was partially supported by NRF grant 2016R1A2B3011647, while Wolf has been supported by the Brain Pool Project of the Korea Federation of Science and Technology Societies (141S-1-3-0022). 


\section{References}

[1] V. I. Arnold and B. A. Khesin, Topological Methods in Hydrodynamics, (1998), Springer-Verlag, New York.

[2] D. Chae, Conditions on the pressure for vanishing velocity in the incompressible fluid flows in $\mathbb{R}^{N}$, Comm. PDE, 37, (2012), pp. 1445-1455.

[3] D. Chae and P. Constantin, Remarks on a Liouville-type theorem for Beltrami flows, Int. Math. Res. Notices, 2015, no.20, (2015), pp. 10012-10016.

[4] P. Constantin and A. Majda, The Beltrami spectrum for incompressible fluid flows, Comm. Math. Phys., 115, no. 3, (1988), pp. 435-456.

[5] C. De Lellis and L. Székelyhidi Jr., Dissipative continuous Euler flows, Invent Math., 193, (2013), pp. 377-407.

[6] A. Enciso and D. Peralta-Salas, Knots and links in steady solutions of the Euler equation, Ann. Math., 175(2), no. 1 (2012), pp. 345-367.

[7] N. Nadirashvili, Liouville theorem for Beltrami flow, Geom. Funct. Anal., 24, (2014), pp. 916-921.

[8] R. Pelz, V. Yakhot and S. A. Orszag, L. Shtilman, and E. Levich, E., Velocityvorticity patterns in turbulent flow, Phys. Rev. Lett., 54, (1985), pp. 2505-2509. 